\title{
Níveis vitamínicos para frangos de corte
}

\author{
Vitamin levels for broiler
}

Ananda Portella Félix ${ }^{I}$ Alex Maiorka ${ }^{I I}$ José Otávio Berti Sorbara ${ }^{\text {III }}$

\section{- REVISÃO BIBLIOGRÁFICA -}

\section{RESUMO}

As vitaminas são micronutrientes que participam de inúmeros processos metabólicos do organismo, sendo, portanto, essenciais para ótima saúde e desempenho do animal. Poucos trabalhos foram realizados nos últimos anos para estimar os melhores níveis de vitaminas para frangos, havendo grande variação entre os níveis empregados comercialmente. Sendo assim, o objetivo deste trabalho é apresentar os conceitos atuais na avaliação das exigências de vitaminas para frangos e os níveis sugeridos pela indústria e pelo meio científico. A maioria dos níveis recomendados pelo NRC (1994) foi baseada em trabalhos antigos, realizados em condições controladas $e$ considerando os níveis mínimos para evitar sinais de deficiência, não avaliando o melhor desempenho nas condições de desafio encontradas a campo. Além disso, as linhagens atuais apresentam maior taxa de crescimento e produção, resultando em maior necessidade nutricional para expressar seu potencial genético. Atualmente, além dos índices zootécnicos, têm se avaliado outros parâmetros na determinação das exigências de vitaminas, tais como imunidade, bem-estar e características da carcaça. Os níveis vitamínicos empregados comercialmente são geralmente superiores aos preconizados pelo NRC (1994), sendo que os níveis das lipossolúveis podem superar até 25 vezes as recomendações e os das hidrossolúveis até cinco vezes. O uso de maiores níveis de vitaminas na dieta de frangos resulta em maior desempenho produtivo e qualidade da carne.

Palavras-chave: exigências nutricionais, frangos, resposta imune.

\section{ABSTRACT}

Vitamins are micronutrients that participe in many metabolic processes of the organism, being essential to animal health and performance. There has been little research conducted over the last years on estimating the best vitamin levels for broilers, having great variation on comercial levels. The objective of this study was to present the actual concepts of the evaluation of vitamin requirements for broiler and the levels recomendated by industry and research centers. The major levels recomendated by NRC (1994) were based on former studies, with controled conditions and considering minimal levels to avoid deficiency signs, not evaluating the best performance on the challenge conditions found in field. Furthermore, modern strains presents greater growth rate and production, resulting in higher nutritional requirements to express their genetical potential. Nowadays, besides zootecnical parameters, it had been evaluating other parameters to determine vitamin requirements, as imunity, well being and carcass characteristics. Comercial vitamin levels used are generally superior than the suggested by NRC (1994), and the liposolubles levels may be higher than 25 times the recomendations and hydrosolubles five times higher. The use of higher vitamin levels in brolier diet results in greater productive performance and meat quality.

Key words: nutritional requirements, broilers, imune response.

\section{INTRODUÇÃO}

As vitaminas são micronutrientes que participam de inúmeros processos metabólicos do organismo, sendo, portanto, essenciais para ótima saúde e desempenho do animal. A deficiência de uma ou mais vitaminas pode levar a distúrbios metabólicos,

IPrograma de Pós-graduação em Ciências Veterinárias, Universidade Federal do Paraná (UFPR), Curitiba, PR, Brasil.

"IDepartamento de Zootecnia, UFPR, Rua dos Funcionários, 1540, Cabral, 80035-050, Curitiba, PR, Brasil. E-mail: amaiorka@ufpr.br. Autor para correspondência.

IIIPrograma de Pós-graduação em Zootecnia, Universidade Estadual de Maringá (UEM), Maringá, PR, Brasil. 
resultando em queda na produtividade, no crescimento e no desenvolvimento de doenças. Já o aumento na suplementação de certas vitaminas tem efeitos positivos, principalmente quanto à imunidade.

A formulação comercial de dietas para frangos de corte consiste na combinação de ingredientes em proporções adequadas para atingir o perfil nutricional desejado, visando nível ótimo entre desempenho e custo e, portanto, máxima rentabilidade. Entre os componentes utilizados na formulação estão os suplementos de micronutrientes, como as vitaminas.

Como a maioria das vitaminas não é sintetizada em quantidades suficientes para atender a demanda fisiológica do organismo, elas devem ser obtidas da dieta e, embora os suplementos vitamínicos correspondam à pequena porcentagem da fórmula $(0,1$ a $0,5 \%$ ), as vitaminas podem representar de $1-3 \%$ do custo da ração, sendo que a $\mathrm{A}$ e $\mathrm{E}$ chegam a corresponder a $50 \%$ do custo total do suplemento vitamínico (TOLEDO et al., 2006).

A literatura apresenta grande variação acerca dos níveis de vitaminas empregados comercialmente para frangos (BASF, 1995; BARROETA et al. 2002; LEESON, 2007). Sendo assim, em virtude das dificuldades em estabelecer os níveis vitamínicos para frangos, a presente revisão teve como objetivo apresentar os conceitos atuais na avaliação das exigências de vitaminas para frangos e os níveis sugeridos pela indústria e pelo meio científico.

\section{Recomendações}

Ao avaliar os níveis de suplementação vitamínica, o nutricionista deve considerar vários fatores que podem exigir mudanças nas exigências, tais como: linhagem, sexo, manejo, idade e estresse (MOREIRA et al., 1998), além de outros fatores ligados à ração, tais como ingredientes, nível de energia, processamento, armazenamento e fontes das vitaminas.

Os níveis de vitaminas sugeridos por órgãos de pesquisa, como National Research Council (NRC), Agriculture and Food Research Council (AFRC), Institut National de Recherche Agronomique (INRA) e“Tabelas brasileiras para aves e suínos” são importantes bases para estimativa dos níveis a serem empregados nas diferentes fases de produção. Entretanto, esses órgãos apresentam apenas as exigências mínimas, as quais geralmente não são suficientes em condições de campo, tendo pouca correlação com os níveis empregados comercialmente.

Vários estudos realizados para determinar as necessidades de vitaminas para frangos foram realizados em condições controladas e/ou com dietas purificadas ou semipurificadas, de alta digestibilidade, sendo compostas por ingredientes pouco usuais na alimentação de frangos, o que também demonstra a baixa correlação com a realidade no campo (LEESON, 2007). Além disso, poucos são os trabalhos conduzidos nos últimos 30 anos para estimar as exigências de vitaminas para frangos, os quais apresentam maior potencial genético de crescimento, com melhora superior a 20\% na conversão alimentar (CA) (PÉREZVENDRELL et al., 2002) e ganho de peso médio diário $87 \%$ superior (de 26,8g dia ${ }^{-1}$ em 1970 a $50 \mathrm{~g} \mathrm{dia}^{-1}$ em 2000) (BARROETA et al., 2002).

Atualmente, novas variáveis, além dos sinais de deficiência e desempenho, estão sendo avaliadas a fim de determinar as exigências de vitaminas para frangos, como resposta imune, bem-estar e enriquecimento de vitaminas na carcaça, visando melhor aspecto, maior tempo de prateleira e valor nutricional da carne para os consumidores.

O emprego de maiores níveis vitamínicos para frangos vem sendo utilizado a fim de compensar variações no consumo, biodisponibilidade das vitaminas da dieta, fatores antiqualitativos dos alimentos, estresse, entre outros. Geralmente respostas significativas do sistema imune ocorrem apenas quando as vitaminas são suplementadas em níveis pelo menos 10 vezes superiores aos do NRC (1994) ou duas a três a vezes maiores que os utilizados comercialmente (LEESON, 2007).

Ao avaliar dois níveis de suplementação vitamínica para frangos, CASTAING et al. (2003) concluíram que o maior nível (dobro do padrão comercial) resultou em crescimento superior aos 38 dias (1.919g) em relação ao nível inferior (1.878g). Além disso, houve maior deposição de vitamina E na carcaça. HERNANDEZ et al. (2002) também encontraram maiores teores de vitaminas no músculo de frangos suplementados com maiores níveis de vitaminas, com efeito mais pronunciado em aves criadas sob maior densidade de alojamento.

Com relação às recomendações de ROSTAGNO et al. (2000 e 2005) nas “Tabelas brasileiras para aves e suínos”, são observadas poucas mudanças quantitativas nos níveis apresentados na 2aㅡ edição em comparação à $1^{\mathrm{a}}$ edição, confirmando o escasso número de pesquisas que determinam as exigências de vitaminas nos últimos anos. Os níveis que sofreram pequeno acréscimo foram os das vitaminas: $\mathrm{E}, \mathrm{B}_{2}$, ácido pantotênico, ácido fólico e colina, para a fase inicial, e E, $\mathrm{B}_{2}$, ácido pantotênico, ácido fólico, colina e $\mathrm{B}_{6}$ para a fase de crescimento, com pequena redução nos níveis de vitamina $\mathrm{K}$ nesta fase. Já para a fase final houve aumento nas recomendações de quase todas as vitaminas, exceto para a colina (redução do nível) e $\mathrm{B}_{12}$ 
(manteve-se o nível). As tabelas 1 e 2 mostram os níveis de vitaminas para as fases inicial e de crescimento, respectivamente, de frangos de corte, segundo recomendações do NRC (1994), ROSTAGNO et al. (2005) e DSM(2006).

Retirada do suplemento vitamínico na fase final

Tendo em vista que a deficiência de vitaminas leva longos períodos para exteriorizar os primeiros sinais clínicos, existe uma tendência de minimizar os níveis ou até mesmo da retirar o suplemento vitamínico da ração final dos frangos, com a alegação de que em dietas práticas (milho e farelo de soja) parte das exigências de vitaminas é suprida. Além disso, vitaminas lipossolúveis são armazenadas nos tecidos adiposos do organismo.

Em uma revisão sobre suplementos vitamínicos comerciais, a BASF (1995) relatou maior coeficiente de variação entre os níveis vitamínicos para a fase final em relação aos das fases pré-inicial e crescimento. O mesmo estudo também relatou que apenas seis dietas para frangos na fase final, de um total de 62 avaliadas, não recebiam suplementação vitamínica, sendo comumente observada a retirada do suplemento três dias antes do abate.

Em experimento realizado por CHRISTMAS et al. (1995), a retirada do suplemento vitamínico durante a última ou as duas últimas semanas de vida até o abate aos 42 dias reduziu o ganho de peso dos frangos, mas não afetou o consumo de ração e a conversão alimentar (CA). Resultados semelhantes foram obtidos por TEETER \& DEYHIM (1998), que também observaram menor ganho de peso e menor peso da carcaça em frangos sem suplementação vitamínica na última semana antes do abate.

Ao avaliar a retirada do suplemento vitamínico na fase final de frangos de corte, MAIORKA et al. (2002) verificaram melhor CA em aves suplementadas com vitaminas até o abate em relação as que foram privadas de suplementação na ração de retirada, entretanto, os autores não encontraram diferença na composição da carcaça. Já MOREIRA et al. (1998) encontraram maior teor de proteínas na carcaça de frangos que receberam vitaminas até o abate contra aqueles privados de suplementação na fase final. PATEL et al. (1997) e DEYHIM et al. (1990) encontraram redução de $43 \%$ e $50 \%$, respectivamente, nos níveis de riboflavina no músculo de frangos privados de suplementação vitamínica na fase final.

Necessidades de vitaminas

Vitaminas lipossolúveis

Os níveis das vitaminas lipossolúveis são os que mais variam entre os preconizados pelo NRC (1994) e os níveis comerciais. Isso ocorre em função de que estas vitaminas estão envolvidas principalmente com o desenvolvimento e a manutenção das estruturas dos tecidos, a imunidade e a melhoria na qualidade da carne e podem ser armazenadas no organismo. Os níveis de vitamina A empregados comercialmente são, em média, cinco a oito vezes superiores aos preconizados pelo NRC (1994), os de vitamina D, 10 a 25 vezes, vitamina $E$, três a 24 vezes e vitamina $K$, quatro a oito vezes superiores.

VitaminaA

A deficiência de vitamina A pode causar efeito depressivo na resposta imune de frangos, como

Tabela 1 - Níveis de vitaminas para fase inicial de frangos (quantidade $\mathrm{kg}^{-1}$ de ração).

\begin{tabular}{llccl}
\hline Vitamina & & NRC (1994) & ROSTAGNO et al. (2005) & DSM (2006) \\
\hline Vitamina $\mathrm{A}$ & UI & 1.500 & 10.000 & $8.000-12.500$ \\
Vitamina $\mathrm{D}_{3}$ & $\mathrm{UI}$ & 200 & 2.000 & $3.000-5.000$ \\
Vitamina E & $\mathrm{UI}$ & 10 & 35 & $150-240$ \\
Vitamina K & $\mathrm{mg}$ & 0,50 & 1,7 & $2-4$ \\
Tiamina $\left(\mathrm{B}_{1}\right)$ & $\mathrm{mg}$ & 1,80 & 1,5 & $2-3$ \\
Riboflavina $\left(\mathrm{B}_{2}\right)$ & $\mathrm{mg}$ & 3,6 & 5 & $7-9$ \\
Piridoxina $\left(\mathrm{B}_{6}\right)$ & $\mathrm{mg}$ & 3,5 & 2,4 & $3-6$ \\
Cianocobalamina $\left(\mathrm{B}_{12}\right)$ & $\mathrm{mg}$ & 0,010 & 0,012 & $0,015-0,040$ \\
Ácido Fólico & $\mathrm{mg}$ & 0,55 & 0,700 & $1,0-2,0$ \\
Ácido Nicotínico & $\mathrm{mg}$ & 35 & 35 & $50-80$ \\
Ácido Pantotênico & $\mathrm{mg}$ & 10 & 12 & $10-18$ \\
Biotina & $\mathrm{mg}$ & 0,15 & 0,070 & $0,15-0,30$ \\
Colina & $\mathrm{mg}$ & 1.300 & 300 & $300-600$ \\
\hline
\end{tabular}

Ciência Rural, v.39, n.2, mar-abr, 2009. 
Tabela 2 - Níveis de vitaminas para fase de crescimento de frangos (quantidade $\mathrm{kg}^{-1}$ de ração).

\begin{tabular}{lllll}
\hline Vitamina & & NRC (1994) & ROSTAGNO et al. (2005) & DSM (2006) \\
\hline Vitamina $\mathrm{A}$ & $\mathrm{UI}$ & 1.500 & 8.000 & $8.000-12.000$ \\
Vitamina $\mathrm{D}_{3}$ & $\mathrm{UI}$ & 200 & 1.600 & $2.000-4.000$ \\
Vitamina $\mathrm{E}$ & $\mathrm{UI}$ & 10 & 28 & $30-50$ \\
Vitamina $\mathrm{K}_{3}$ & $\mathrm{mg}$ & 0,50 & 1,4 & $2-4$ \\
Tiamina $\left(\mathrm{B}_{1}\right)$ & $\mathrm{mg}$ & 1,80 & 1,2 & $2-3$ \\
Riboflavina $\left(\mathrm{B}_{2}\right)$ & $\mathrm{mg}$ & 3,6 & 4 & $5-8$ \\
Piridoxina $\left(\mathrm{B}_{6}\right)$ & $\mathrm{mg}$ & 3,5 & 1,9 & $4-6$ \\
Cobalamina $\left(\mathrm{B}_{12}\right)$ & $\mathrm{mg}$ & 0,010 & 0,010 & $0,020-0,030$ \\
Ácido Fólico & $\mathrm{mg}$ & 0,55 & 0,560 & $1-2$ \\
Niacina & $\mathrm{mg}$ & 30 & 28 & $40-80$ \\
Ácido Pantotênico & $\mathrm{mg}$ & 10 & 9,6 & $10-15$ \\
Biotina & $\mathrm{mg}$ & 0,15 & 0,056 & $0,15-0,30$ \\
Colina & $\mathrm{mg}$ & 1.000 & 240 & $300-500$ \\
\hline
\end{tabular}

demonstrado por DALLOU et al. (2002) e FRIEDMAN et al. (1991), os quais relataram menor resposta imune à infecção de Eimeria acervulina e Escherichia coli, respectivamente, em frangos alimentados com dietas deficientes em vitamina A. Em contrapartida, o excesso de vitamina $\mathrm{A}$ pode causar efeito antagônico às vitaminas $\mathrm{E}_{\mathrm{e}} \mathrm{D}_{3}$.

Em estudo com frangos de corte, SURAI (2000) relatou que níveis de vitamina A superiores a 10200 vezes os comerciais causaram diminuição na concentração de vitamina E no sangue e um acúmulo de vitamina A no fígado de 100 vezes o valor fisiológico normal. Em relação à disponibilidade de vitamina $\mathrm{D}_{3}$, BRITTON (1992) verificou menor teor de matéria mineral na tíbia de frangos alimentados com o maior teor de vitamina A e sem colecalciferol.

Vários autores que analisaram diferentes níveis de vitamina A na dieta, como BRITTON (1992) (5.000, 50.000 e $150.000 \mathrm{UI} \mathrm{kg}^{-1}$ ), TOLEDO et al. (2006) (5.000, 10.000 e 15.000UI kg-1) e WYATT (1991) (6.600, 19.800, 39.600 e $79.200 \mathrm{UI} \mathrm{kg}^{-1}$ ), não encontraram diferença no desempenho de frangos. Estes resultados confirmam a dificuldade em estabelecer níveis de vitaminas para frangos criados comercialmente, já que os desafios de campo não são reproduzidos na maioria dos experimentos, o que pode subestimar as necessidades das aves.

Vitamina E

A importância da vitamina E na resposta imune foi observada por KONJUFCA et al. (2004), os quais relataram maior atividade fagocítária dos macrófagos de frangos suplementados com 110 e $220 \mathrm{mg}$ de vitamina $E \mathrm{~kg}^{-1}$ em relação ao controle (16 $\mathrm{mg} \mathrm{kg}^{-1}$ ).

Suplementando 200mg de vitamina $\mathrm{E} \mathrm{kg}^{-1}$ da dieta, FRIGG (1990) encontrou maior ganho de peso e de CA, quando comparados a frangos que receberam 25mg. Já SELL et al. (1994), suplementando de 0 a $300 U I$ $\mathrm{kg}^{-1}$, e TOLEDO et al. (2006), utilizando 10, 20 e 30mg de vitamina $\mathrm{E} \mathrm{kg}^{-1}$ da dieta, não observaram diferença no desempenho dos frangos. Para frangos de corte sexados, BLUM et al. (1992) verificaram melhores taxas de crescimento em machos suplementados com 40 e 80mg de vitamina $\mathrm{E} \mathrm{kg}^{-1}$ da dieta, enquanto as fêmeas não apresentaram diferença quanto ao ganho de peso.

O teor de ácidos graxos poliinsaturados (PUFA) da dieta afeta o depósito de vitamina E nos tecidos, como demonstrado por BARROETA(2007), o qual observou diminuição no teor de a-tocoferol na coxa (menos $0,42 \mathrm{mg} \mathrm{kg}^{-1}$ ) com o aumento nos níveis dietéticos de PUFA (1 $\mathrm{g} \mathrm{kg}^{-1}$ ) em dietas com 200mg de vitamina $\mathrm{E} \mathrm{kg}^{-1}$. Segundo o autor, há alguma destruição do a-tocoferol devido ao alto teor de PUFA e, portanto, as exigências de vitamina $E$ aumentam de 2,5 a 3,7 $\mathrm{mg} \mathrm{g}^{-1}$ de PUFA dietético. CORTINA et al. (2005) concluíram que a suplementação com até $200 \mathrm{mg} \mathrm{kg}^{-1}$ de a-tocoferol previne até $88 \%$ da máxima oxidação lipídica, no entanto, níveis superiores a este não melhoraram a estabilidade da carne de coxa de frangos. Resultados semelhantes foram obtidos por BARTOV \& FRIGG (1992), os quais encontraram maior estabilidade da carne de frangos suplementados com 150mg de a-tocoferol kg-1 durante todo o ciclo produtivo, em comparação com os suplementados apenas nas últimas duas semanas. SHEEHY et al. (1994) indicaram melhoria no sabor de carnes estocadas a $4^{\circ} \mathrm{C}$ derivadas de frangos suplementados com $160 \mathrm{UI}$ de vitamina $\mathrm{E} \mathrm{kg}^{-1}$, comparados com os que receberam apenas 20UI de vitamina $\mathrm{E} \mathrm{kg}^{-1}$. Segundo LEESON (2007), geralmente a suplementação de 100-400UI de vitamina $\mathrm{E} \mathrm{kg}^{-1}$ de dieta tem demonstrado que promove a qualidade da carne na maioria dos estudos.

Ciência Rural, v.39, n.2, mar-abr, 2009. 
Vitamina $\mathrm{D}_{3}$

A importância dos raios ultravioletas na síntese de vitamina $\mathrm{D}_{3}$ em frangos foi estudada por EDWARDS et al. (1994), os quais concluíram que o dobro (400UI kg-1) do nível recomendado pelo NRC (1994) fornecido para aves privadas de luz solar não foi suficiente para permitir ganho de peso e mineralização óssea igual às aves que receberam iluminação solar. Além disso, frangos privados de sol, que receberam 1.600UI kg-1 de colecalciferol, apresentaram desempenho comparável aos frangos que dispunham de luz solar.

Estudos mostram que apenas altos níveis de vitamina $\mathrm{D}_{3}\left(2.250-15.000 \mathrm{UI} \mathrm{kg}^{-1}\right)$ diminuíram a incidência de anomalias ósseas em pintos (EDWARDS et al., 1992; SILVA et al., 2001; WHITEHEAD et al., 2004). Em relação à importância da vitamina $D_{3}$ no sistema imune, ASLAM et al. (1998) relataram redução na atividade dos macrófagos de frangos sem suplementação de vitamina $\mathrm{D}_{3}$, não havendo diferença quanto à resposta imune de frangos suplementados com 2.000 a $4.000 \mathrm{UI} \mathrm{D}_{3} \mathrm{~kg}^{-1}$ da dieta, como empregado comercialmente.

Vitamina K

A vitamina $K$ é sintetizada por microrganismos no intestino, no entanto, seu aproveitamento pelas aves é muito limitado, principalmente em casos de administração prolongada de antibióticos e/ou presença de micotoxinas na dieta.

Ao avaliar os efeitos de níveis crescentes de vitamina $\mathrm{K}$ (menadiona) no desenvolvimento ósseo de frangos, ZHANG et al. (2003) obtiveram melhores resultados com a suplementação de $8 \mathrm{mg} \mathrm{kg}^{-1}$ na fase inicial e $2 \mathrm{mg} \mathrm{kg}^{-1}$ nas fases de crescimento e terminação. Estes valores se enquadram aos empregados comercialmente, embora sejam quatro a 16 vezes superiores ao sugerido pelo NRC (1994). No entanto, cuidado especial deve ser tomado na fonte de vitamina $\mathrm{K}$ a ser utilizada por ser uma das vitaminas mais sensíveis aos processos de peletização e extrusão.

Vitaminas hidrossolúveis

As hidrossolúveis participam das vias metabólicas dos carboidratos, das proteínas e dos lipídeos, atuando, principalmente, como co-fatores nas reações. A maioria é necessária em menores quantidades que as vitaminas lipossolúveis.

A variação encontrada entre as recomendações do NRC (1994) e os níveis empregados comercialmente é menor que nas vitaminas lipossolúveis, não superando, geralmente, mais que cinco vezes. Entretanto, embora esta variação seja menor, ainda são muitas as contradições encontradas na literatura sobre os níveis ótimos a serem utilizados a campo.

Tiamina (B )

As atuais linhagens podem ter maior necessidade de $\mathrm{B}$ devido à elevada taxa de crescimento, sendo que a suâ deficiência está associada com a síndrome da morte súbita. OLKOWISK \& CLASSEN (1999) concluíram que a suplementação com $2 \mathrm{mg}$ de tiamina $\mathrm{kg}^{-1}$ resultou em maior ganho de peso e conversão alimentar de pintos, enquanto que maiores níveis (8 e 32mg kg-1) diminuíram estes índices. Já matrizes que receberam maiores níveis (8 e 32mg de $\mathrm{B}$ $\mathrm{kg}^{-1}$ da dieta) obtiveram pintos com maior ganho de peso e CA, mostrando a importância da suplementação das matrizes no desempenho da progênie.

Ao avaliar dois níveis de $\mathrm{B}_{1}(1,4 \mathrm{mg}$ e 3,0mg $\mathrm{kg}^{-1}$ ), PÉREZ-VENDRELL et al. (2002) encontraram maior deposição de $B_{1}\left(0,072 \mathrm{mg} 100 \mathrm{~g}^{-1}\right)$ no peito de frangos suplementados com $3,0 \mathrm{mg} \mathrm{kg}^{-1}$ da dieta que em frangos suplementados com $1,4 \mathrm{mg} \mathrm{kg}^{-1}(0,056 \mathrm{mg}$ $100 \mathrm{~g}^{-1}$ de peito).

Riboflavina $\left(\mathrm{B}_{2}\right)$

A deficiência de $B_{2}$ é uma das carências mais prováveis a campo, já que dietas com milho e farelo de soja fornecem de 2,0 a 2,6mg $\mathrm{B}_{2} \mathrm{~kg}^{-1}$, valores estes abaixo dos níveis mínimos preconizados pelo NRC (1994) (3,0-3,6mg B $\mathrm{kg}^{-1}$ ) (BARROETA et al., 2002). Segundo RUTZ et al. (1998), houve melhor desempenho em frangos de corte suplementados com 4,0mg de riboflavina, enquanto que OLKOWISK \& CLASSEN (1998) encontraram maior taxa de crescimento das aves alimentadas com 5,0mg de $\mathrm{B}_{2} \mathrm{~kg}^{-1}$ da dieta.

\section{Ácido Nicotínico}

Ao avaliar dois níveis de niacina para frangos na fase inicial (15 e 22,5 $\mathrm{mg} \mathrm{kg}^{-1}$ ), crescimento (12,50 e 18,75mg) e final (9,99 e 15mg), SANTIN et al. (2000) não encontraram diferença no desempenho. Já RUIZ et al. (1990) observaram que frangos suplementados com $32 \mathrm{mg} \mathrm{kg}^{-1}$ da dieta apresentaram maior desempenho e menores casos de deformidades de pernas em relação às aves sem suplementação.

Certas situações de estresse exigem maior demanda energética do organismo e como a nicotinamida forma parte das co-enzimas NADH e NADPH é provável que maiores níveis de niacina nestas condições possam contribuir com melhor desempenho.

Ciência Rural, v.39, n.2, mar-abr, 2009. 


\section{Ácido Pantotênico (B $\mathrm{B}_{5}$ )}

Ao avaliarem níveis de $\mathrm{B}_{5}$ duas e quatro vezes superiores às recomendações do NRC (1994), DEYHIM et al. (1990) não obtiveram diferença no desempenho das aves, no entanto, houve aumento na deposição de ácido pantotênico na carne de 35\% e $74 \%$, respectivamente. Resultados semelhantes foram obtidos por PÉREZ-VENDRELL et al. (2002), os quais também verificaram maior depósito de ácido pantotênico (1,63 x 1,16mg $100 \mathrm{~g}^{-1}$ de peito) em aves que receberam maior nível na dieta $\left(15,0 \times 10,4 \mathrm{mg} \mathrm{kg}^{-1}\right.$, respectivamente).

\section{Piridoxina $\left(\mathrm{B}_{6}\right)$}

As exigências de $B_{6}$ são maiores em dietas com elevados níveis de proteína e, portanto, sua deficiência reduz a retenção de nitrogênio (BARROETA et al, 2002). Os níveis suplementados comercialmente (3,0-6,0 $\left.\mathrm{mg} \mathrm{kg}^{-1}\right)$ não variam muito em relação aos sugeridos pelo NRC (1994) (3,0-3,5 $\left.\mathrm{mg} \mathrm{kg}^{-1}\right)$, entretanto, BEIRNE \& JENSEN (1981) verificaram diminuição de problemas de pernas com níveis maiores de piridoxina na dieta.

\section{Biotina}

Ao estudar o efeito de níveis de biotina no desempenho de frangos de corte, OLOYO \& ORGUNMODEDE (1991) verificaram que a exigência mínima é de $0,12 \mathrm{mg} \mathrm{kg}^{-1}$, enquanto que o nível de $0,16 \mathrm{mg}$ $\mathrm{kg}^{-1}$ foi necessário para prevenir dermatite, síndrome do fígado, rins gordurosos (FLKS) e deformidades de pernas, além de reduzir a mortalidade. Já STEELE et al. (1982) não encontraram diferença significativa na suplementação de 0,20 ou $1,0 \mathrm{mg}$ de biotina dia $^{-1}$ na água de bebida quanto à mortalidade e ao desempenho.

\section{Ácido Fólico}

Ao comparar dietas com e sem adição de ácido fólico para aves, PESTI \& ROWLAND (1991) concluíram que o crescimento dos frangos aumentou significativamente de zero a 18 dias com a suplementação de $1,52 \mathrm{mg} \mathrm{kg}^{-1}$ de folacina. Já SANTIN et al. (2000), trabalhando com dois níveis de ácido fólico: fase inicial (0,8 e 1,2 $\left.\mathrm{mg} \mathrm{kg}^{-1}\right)$, crescimento (0,66 e 1,00mg) e final (0,53 e 0,80mg), não encontraram diferença no desempenho.

As necessidades de ácido fólico aumentam em dietas com maiores níveis protéicos e se as quantidades de $\mathrm{B}_{12}$ e colina são insuficientes. Sendo assim, com níveis adequados de metionina e colina, estudos sugerem a suplementação com 1,2-1,4mg de ácido fólico $\mathrm{kg}^{-1}$ da dieta (BARROETA et al., 2002).
Cianocobalamina $\left(\mathrm{B}_{12}\right)$

As necessidades de vitamina $B$ são muito pequenas comparadas com outras vitaminnas, sendo que os níveis utilizados atualmente variam entre 0,010 0,040 $\mathrm{mg} \mathrm{kg}^{-1}$. Segundo PORSTMOUTH (1996), citado por BARROETA (2002), dietas sem inclusão de farinhas de origem animal devem ter o suplemento acrescido de $20 \%$ desta vitamina, considerando exigência de $0,012 \mathrm{mg} \mathrm{kg}^{-1}$ da dieta.

\section{Vitamina C}

Vários estudos mostram efeito benéfico da suplementação de vitamina $C$ na dieta apenas em situações de estresse. VOLKER \& WEISER (1993) observaram maior taxa de crescimento e maior ganho de peso de pintos suplementados com 100mg de vitamina $\mathrm{C} \mathrm{kg}^{-1}$ da dieta. Os autores também citam um efeito sinérgico entre a vitamina $\mathrm{C}$ e a $\mathrm{D}_{3}$, já que as aves suplementadas com vitamina $C$ apresentaram melhor formação do esqueleto.

A vitamina $C$ também desempenha importante função antioxidante na restauração da vitamina $\mathrm{E}$ por meio da redução dos radicais tocoferoxil (BARROETA et al., 2002). BOU et al. (2001) concluíram que a suplementação com $110 \mathrm{mg}$ de vitamina $C$ diminuiu a oxidação da carne e melhorou sua qualidade sensorial.

Segundo BARROETA et al. (2002), a suplementação com 200-300ppm de ácido ascórbico tem mostrado bons resultados a campo, enquanto que, em situações extremas de estresse, podem ser empregados níveis de 500-1.000ppm. Entretanto, observa-se grande variabilidade nas respostas experimentais obtidas em virtude da baixa estabilidade desta vitamina, das doses empregadas e da duração, da idade das aves e da intensidade dos agentes estressores.

Colina

A colina é um aminoálcool quaternário essencial para a síntese de fosfolipídeos, como a lecitina e a esfingomielina. As exigências de colina podem ser influenciadas pelo teor de lipídeos da dieta (maiores níveis de colina em dietas ricas em lipídeos) e aminoácidos sulfurados, já que é sintetizada no fígado a partir da metionina (BARROETA et al., 2002).

Ao trabalhar com a adição de $1.000 \mathrm{mg} \mathrm{kg}^{-1}$ de colina, $1.000 \mathrm{mg} \mathrm{kg}^{-1}$ de betaína ou a combinação de $500 \mathrm{mg} \mathrm{kg}^{-1}$ de betaina mais $500 \mathrm{mg}$ de colina, WALDROUP et al. (2006) observaram maior produção de peito em frangos, independentemente dos níveis de metionina. Em função desta inter-relação entre a colina, a metionina e a betaína, torna-se difícil estabelecer as 
exigências de colina, justificando as variações encontradas entre os estudos. Já HASSAN et al. (2005) encontraram melhor desempenho de frangos suplementados com $1.172 \mathrm{mg} \mathrm{kg}^{-1}$ de colina ou com a combinação de $872 \mathrm{mg} \mathrm{kg}^{-1}$ de colina e $0,072 \%$ de betaína.

Segundo o NRC (1994), as exigências de colina variam entre 750 e $1.300 \mathrm{mg} \mathrm{kg}^{-1}$ de alimento, dependendo da idade e da linhagem. Comercialmente, os níveis de colina variam de 1200 a $1800 \mathrm{mg} \mathrm{kg}^{-1}$, com tendência de aumento dos níveis na dieta inicial e de crescimento.

\section{CONCLUSÕES}

A suplementação vitamínica com níveis superiores às recomendações mínimas do NRC e de demais centros de pesquisa tem resultado em melhoria no desempenho zootécnico dos frangos de corte. Entretanto, novos experimentos são necessários quanto ao estudo das exigências de vitaminas das linhagens contemporâneas, uma vez que há escassez de dados atuais e muitas dúvidas quanto aos melhores níveis vitamínicos que devem ser suplementados. Nesse contexto, além dos índices zootécnicos, há importantes variáveis a serem analisadas, tais como imunologia, interação entre vitaminas e outros nutrientes, composição, qualidade da carne e segurança alimentar, desempenho sob condições de estresse e bem-estar.

\section{REFERÊNCIAS}

ASLAM, S.M. et al. Vitamin D deficiency alters the immune response of broiler chicks. Poultry Science, v.77, p.842849, 1998.

BARROETA, A.C. et al. Óptima nutrición vitamínica de los animales para la producción de alimentos de calidad. Barcelona: Pulso ediciones, 2002. 208p.

BARROETA, A.C. Nutritive value of poultry meat: relationship between vitamin E and PUFA. World's Poultry Science Journal, v. 63, p.267-284, 2007.

BARTOV, I.; FRIGG, M. Effect of high concentrations of dietary vitamin $\mathrm{E}$ during various age periods on performance, plasma vitamin $\mathrm{E}$ and meat stability of broiler chicks at 7 weeks of age. British Poultry Science, v.33, p.393-402, 1992.

BASF CORPORATION. Optimum vitamin supplementation in poultry: laboratory or commercial field requirements, 1995. Capturado em 12 nov. 2007. Online. Disponível na Internet: http://www.basf.br

BEIRNE, M. J.; JANSEN, L. S. Influence of high levels of piridoxyne on twisted legs in broilers. Poultry Science, v.60, p.1026-1029, 1981.
BLUM, J.C. Effect of dietary vitamin E supplie in broilers, male and female growth rate, viability, imune response, fat content and meat flavour variations during storage. Arch Geflugelkd, v.56, p.37-42, 1992.

BOU, R. et al. Influence of dietary fat source, alpha-tocopherol, and ascorbic acid supplementation on sensory quality of chicken meat. Poultry Science, v.80, p.800-807, 2001.

BRITTON, W.M. Dietary vitamin A effect on broiler chick cholecalciferol requirement. Poultry Science, v.71, p.328334, 1992

CASTAING, J. et al. Influencia de la utilización de dos niveles de vitaminas sobre los resultados productivos de broilers. Producción animal, v.18, n.187, p.54-62

CORTINA, L. et al. Influence of the dietary polyunsaturation level on chicken meat quality: Lipid oxidation. Poultry Science, v.84, p.48-55, 2005.

CHRISTMAS, R.B. et al. The absence of vitamins and trace minerals on broiler performance. Journal of Applied Poultry Research, v.4, n.3, p.407-410, 1995.

DALLOUL, R.A. et al. Effect of vitamin A deficiency on host intestinal immune response to Eimeria acervulina in broiler chickens. Poultry Science, v.81, p.1509-1515, 2002.

DEYHIM, F. et al. Riboflavin and pantothenic acid requirement of broilers through eight weeks posthatching. Poultry Science, v.129, p.195-201, 1990.

EDWARDS JR., H.M. et al. Effects of dietary calcium on tibial dyschondroplasia. Interaction with light, cholecalciferol, 1,25dihydroxycholecalciferol, protein, and synthetic zeolite. Poultry Science, n.12, p.2041-2055, 1992.

EDWARDS JR. et al. Quantitative requirement for cholecalciferol in the absense of ultraviolet light. Poultry Science, v.73, p.288-294, 1994

FRIEDMAN, A. et al. Decreased resistance and immune response to Escherichia coli infection in chicks with low or high intakes of vitamin A. Journal of Nutrition. v.121, n.3, p.395-400, 1991.

FRIGG, M. Effects of dietary vitamin E supplies in broilers. Evaluation of parameters related to oxidative stability of broiler meat. Nutrition Abstract, v.22, p.24-30, 1990.

HASSAN, R.A. et al. Growth, carcass quality and serum constituents of slow growing chicks as affected by betaine addition to diets containing different levels of choline. International Journal of Poultry Science, v.4, n.11, p.840850,2005

HERNANDEZ, J.M. et al. Effect of vitamin level in broiler diets on productive parameters and meat deposition. In: EUROPEAN POULTRY CONFERENCE, 11., 2002, Bremen. Anais... Bremen: WPSA, 2002. CD-ROM.

KONJUFCA, V.K. et al. Influence of dietary vitamin E on phagocitic functions of macrophages in broilers. Poultry Science. v.83, p.1530-1534, 2004. 
LEESON, S.; SUMMERS, J.D. Comercial poultry nutrition. Guelp, Ontario, Cananda: University Book, 2005. 355p.

LEESON, S. Vitamin requirements: is there basis for reevaluating dietary specifications? World's Poultry Science Journal, v.63, n.2, p.255-266, 2007.

MAIORKA, A. et al. Dietary vitamin or mineral mix removal during the finisher period on broiler chicken performance. Poultry Science Association, v.11, p.122-126, 2002.

MOREIRA, R.S.R. et al. Efeito da restrição de vitaminas e minerais na alimentação de frangos de corte sobre o rendimento e a composição da carne. Ciência e tecnologia de alimentos, v.18. p.77-81, 1998.

NATIONAL RESEARCH COUNCIL - NRC. Nutrient requirements of poultry. 9.ed. Washington, DC., 1994. $155 p$.

OLKOWSKI, A.A.; CLASSEN, H.L. The study of riboflavin requirement in broiler chickens. International Journal of Vitamin Nutrition Research, n.68, p.316-327, 1998.

OLKOWSKI, A.A.; CLASSEN, H.L. The effects of maternal thiamine nutrition on thiamine status of the offspring in broiler chickens. International Journal of Vitamin Nutrition Research, v.69, p.32-40, 1999.

OLOYO, R.A.; ORGUNMODEDE, B.K. The biotin requirement of broilers feed maize-palm kernel based ration. Beitr Trop Landwirtsch Veterinarmed, v.29, n.2, p.223-233, 1991.

PATEL, K.P. et al. Removal of vitamin and trace mineral supplements from broiler finisher diets. Journal of Apllied Poultry Research, v.6, p.191-198, 1997.

PÉREZ-VENDRELL, A. M. et al. Effect of optimum vitamin levels in broiler diets on performance and meat quality parameters. 2002. Capturado em 30 set. 2007. Online. Disponível na Internet: http://www.dsm.com

PESTI, G.M.; ROWLAND, G.N.I. Folate deficiency in chicks fed diets containing practical ingrediente. Poultry Science, v.70, p.600-604, 1991.

ROSTAGNO, H.S. et al. Tabelas brasileiras para aves e suínos: composição de alimentos e exigências nutricionais. Viçosa, MG: Universidade Federal de Viçosa, 2000. 138p.

ROSTAGNO, H.S. et al. Tabelas brasileiras para aves e suínos: composição de alimentos e exigências nutricionais. 2.ed. Viçosa, MG: Universidade Federal de Viçosa, 2005. 186p.

RUIZ, N. et al. Niacin requirement of broiler chickens fed a corn-soybean meal diet from 1 to 21 days of age. Poultry Science, v.69, p.433-439, 1990.
RUTZ, F. et al. Repleção com riboflavina em pintos Leghorn depletados neste nutriente. Revista Brasileira de Agrociência, v.4, n.3, p.197-200, 1998.

SANTIN, E. et al. Diferentes níveis de ácido fólico e nicotínico em dietas para frangos de corte. Ciência Rural, v.30, n.4, p.681-685, 2000.

SELL, J. et al. Influence of dietary supplementation with vitamin $\mathrm{E}$ and Ascorbic Acid on vitamin E status of poultry. Poultry Science, v.73, n.1, p.13, 1994.

SHEEHY, P.J.A. et al. Advances in research and application of vitamin $\mathrm{E}$ and antioxidant for poultry meat. In: EUROPEAN SYMPOSIUM ON THE QUALITY OF POULTRY MEAT, 12; 1994, Zaragoza. Anais... Zaragoza: Organising Committee 12. th, 1994. p.425-436.

SILVA, F.A. et al. Efeitos do ácido L-glutâmico e da vitamina D no desempenho e anomalias ósseas de pintos de corte. Rẻvista Brasileira de Zootecnia, v.30, p.2059-2066, 2001.

STEELE, P. et al. Effect of biotin supplementation on incidence of acute death syndrome in broiler chickens. Poultry Science, v.61, n.5, p.909-913, 1982.

SURAI, P.F. Effect of selenium and vitamin E of the maternal diet on the antioxidant system of the yolk and the developing chick. Brazilian Poultry Science, v.41, p.235-243, 2000.

TEETER, R.G.; DEYHIM, F. Vitamin withdrawal effects on performance, carcass composition, and tissue vitamin concentration of broilers exposed to various stress types. 1998. Capturado em set. 2007. Online. Disponível na internet: http://www.basf.com

TOLEDO,G.S. et al. Níveis das vitaminas A e E em dietas de frangos de corte de 1 a 42 dias de idade. Ciência Rural, v.36, n.2, p.624-629, 2006.

VOLKER, L.F.; WEISER, H. The relevance of vitamin D e C for bone metabolism in poultry. In: MARYLAND NUTRITION CONFERENCE, 1993, Baltimore. Anais... Baltimore: University of Maryland, 1993. p.42-54.

WALDROUP, P.W. et al. Effects of betaine and choline on response to methionine supplementation in broiler diets formulated to industry standards. Journal of Applied Poultry Research, v.15, p.58-71, 2006.

WYATT, L.C. Effect of high levels of vitamin A supplementation, on skin pigmentation and growth performance in broiler chicks. Poultry Science, v.1, p.134-143, 1991.

WHITEHEAD, C.C. et al. The maximum legal limit for vitamin $\mathrm{D}$ in broiler diets may need to be increased. British Poultry Sçience, v.45, p.24-26, 2004.

ZHANG, C. et al. Effects of dietary vitamin K levels on bone quality in broilers. Archives of Animal Nutrition, v.57, n.3, p.197-206, 2003. 